\title{
Efficient Routing and Spectrum Allocation Considering QoT in Elastic Optical Network
}

\author{
Bingbing Li and Young-Chon Kim
}

\begin{abstract}
In recent years, Orthogonal Frequency Division Multiplexing (OFDM)-based elastic optical network (EON) has drawn increasing attention in both academia and industry. Compared to traditional optical Wavelength Division Multiplexing (WDM) network, EON is capable of supporting variable data rate, accommodating traffic demands flexibly with finer provisioning granularity, and utilizing spectrum more efficiently. Accordingly, the resource management in EON is different from that in WDM networks and addresses new challenges in network design and control. In this paper, we study the routing, modulation level and spectrum allocation (RMLSA) problem in EON. We propose an efficient link-based mixed integer linear programming (MILP) model with the objective to optimally utilize the spectrum resources while considering the constraint on Quality of Transmission (QoT). Then, the performance of the proposed model is evaluated and analyzed through case study.
\end{abstract}

Keywords - Elastic optical network, link-based model, MILP, RMLSA, QoT.

\section{INTRODUCTION}

With the exponential growth of end users and the emergence of bandwidth intensive applications, Internet traffic has been increasing sharply during last decades. The compound annual growth rate (CAGR) of IP traffic in backbone networks is estimated to be kept at $24 \sim 53 \%$ in the near future [1]. To meet the demand, optical Wavelength Division Multiplexing (WDM) network has been widely deployed for Internet backbone and the service providers keep on enlarging network capacity and expanding network size. The bandwidth of one wavelength has been continuously improved to $10 \mathrm{Gbps}, 40 \mathrm{Gbps}$, and nowadays, 100 Gbps. However, traditional WDM networks adopt the ITU-T fixed-grid standard which divides the spectrum of Cband into fixed $50 \mathrm{GHz}$ frequency slots (FSs) [2]. Data rate of $400 \mathrm{Gbps}$ or higher for one wavelength channel cannot be achieved by the fixed grid and modulation format based on existing standard. On the other hand, the bandwidth demands among various applications show high

Manuscript received November 15, 2015; revised January 30, 2016 This work was supported by Basic Research Program through the National Research Foundation of Korea (NRF) funded by the Ministry of Education (2014-055177).

Bingbing $\mathrm{Li}$ is with the Department of Information Technology, Chonbuk National University, Jeonju, Jeonbuk, Korea (e-mail: bingbingli@jbnu.ac.kr).

Young-Chon Kim is with the Department of Information Technology, Chonbuk National University, Jeonju, Jeonbuk, Korea (corresponding author, phone: +82)63-271-2413; fax: +82)63-271-2413; e-mail: yckim@jbnu.ac.kr).

doi: $10.11601 /$ ijates.v5i1.135 heterogeneity. Current optical network operating on the fixed grid has to allocate a complete wavelength even when required bandwidth is much smaller than the wavelength capacity, leading to inefficient utilization of spectrum resources. The support for $400 \mathrm{Gbps}, 1 \mathrm{Tbps}$, and other high bit rate demands and the flexibility to accommodate heterogeneous demands drive us to develop a new paradigm which can overcome the shortages of WDM networks.

Recently, the Elastic Optical Network (EON) has been proposed. The main advantages of EON are: (1) bandwidth allocation based on finer granularity of a subcarrier rather than a wavelength; (2) ability to dynamically adapt channel date rate to required demand via bandwidth-variable transceivers (BVT) and bandwidth-variable optical crossconnect (BV-OXC); (3) high spectrum efficiency by flexibly adjusting modulation format via software. Among all enabling technology to realize EON architecture, optical Orthogonal Frequency Division Multiplexing (OFDM) has become the focus of extensive research effort. OFDM is a multi-carrier modulation technique by which the data flow is distributed over an arbitrary set of orthogonal low data rate subcarriers. These orthogonal subcarriers overlap in the frequency domain, leading to flexible resource allocation and efficient utilization of the spectrum resources. Comparing to traditional WDM networks, OFDM-based EON can achieve sub-wavelength and super-wavelength accommodation for various traffic demands. By allocating one or multiple contiguous FSs with appropriate modulation format, a spectrum path can be all-optically established between the source and destination nodes. If two spectrum paths share one or more common physical links, these spectrum paths should be separated by guard band for filtering and recovering signal. As the fundamental issue in EON, the Routing and Spectrum Allocation (RSA) problem should be taken into account, which is different from and more complex than the traditional Routing and Wavelength Assignment (RWA) problem in WDM networks.

In OFDM-based EON, each subcarrier can be processed individually through digital signal processing (DSP) implemented at both the transmitting and the receiving ends. That is, the modulation format can be adjusted according to the traffic demand and the transmission distance of optical path. Due to the accumulated signal impairment on the consecutive fiber links along the optical path, the maximum optical transmission distance (called transmission reach) is severely limited when the quality of transmission (QoT) is considered. Although optical OFDM can support significant higher channel capacity, the choice of the modulation format has to take into account the QoT. The relationship between 
the capacity and transmission reach is given in [3]: an extra bit can be added per symbol, and the transmission reach should be halved for every $3 \mathrm{~dB}$ gain in signal-to-noise ratio (SNR). For example, modulation format 8QAM (3 bit/symbol) can be used instead of QPSK (2 bit/symbol), while the transmission reach of 8QAM signal should be reduced to half the transmission reach of QPSK. The consideration of QoT poses additional challenges on RSA: decision of appropriate modulation level.

To address all the issues mentioned above, new strategies and algorithms for Routing, Modulation Level and Spectrum Allocation (RMLSA) have to be developed. The challenges in new spectrum management schemes are:

- Spectrum contiguity constraint: The spectrum allocated to a request must be a block of contiguous FSs.

- Spectrum continuity constraint: This constraint is similar to the wavelength continuity constraint in WDM networks. The same contiguous FSs must be allocated on the consecutive links along the optical end-to-end path of a request.

- Decision of modulation level: The choice of modulation level is affected by required bandwidth and the limitation on transmission reach.

In this paper, we study the RMLSA problem in transparent EON. An efficient link-based MILP formulation is proposed to determine the routing, modulation level and spectrum allocation to each traffic demand with the consideration of QoT. To improve the spectrum efficiency, it tends to choose highest modulation level. However, the transmission reach of the optical path with the highest modulation level is very limited if acceptable QoT is considered. Hence, the proposed model needs to find the tradeoff among the number of required FSs, modulation level, and transmission reach. Our objective is to minimize the number of required FSs in network to accommodate all traffic demands.

The rest of this paper is organized as follows: In section II, the mathematical model is presented and explained. In section III, the MILP models are evaluated and compared by illustrative examples; and the numerical results will be analyzed. Finally, we conclude the paper in Section IV.

\section{RELATED WORK}

The RMLSA problem has been studied in several literatures. Reference [4, 5] introduced a path-based ILP formulation with the objective to minimize the maximum utilized spectrum slot index assigned on any link in the network. This work considered pre-computed $k$-shortest paths for each $(s, d)$ pair. The constraints included: starting frequency ordering, non-overlapping spectrum allocation constraints. This model can solve routing and spectrum allocation jointly. Then, the same authors presented a method to decompose RSA into two formulations which address the routing (R) and spectrum assignment (SA) sub-problems respectively in [4]. A set of paths for each demand are predetermined as the input to $\mathrm{R}$ formulation. By solving the first formulation, one path per demand can be determined in order to minimize the amount of traffic flow (i.e., spectrum use) on any link. Then, the SA formulation assigns FSs to demands so as to minimize the maximum FS index. Although solving each sub-problem separately and sequentially is more scalable than the joint ILP formulation, the joint optimal solution of the RSA problem might not be guaranteed. In [5], modulation level is considered and the RSA is decomposed into RML and SA sub-problems, which are solved sequentially. The choice of the modulation level takes into account the QoT of the connection. Due to the pre-calculation of candidate paths, the candidate modulation levels are also limited. Reference [6] proposed an ILP model with the objective to minimize total network cost. The cost is defined proportional to the total edge length and data rate of the traffic flow. And the model includes the physical capacity on each optical fiber and the traffic bounds constraints. Reference [7] proposed an MILP formulation to minimize total network energy consumption. The authors define the energy model which considers the energy cost of transponder, FS, amplifier and electronic processing. The network architecture is assumed to be the multi-hop grooming scenario. The routes of lightpaths are precomputed and given as input parameters. The formulation includes the wavelength continuity, capacity, and maximum subcarrier constraints. In particular, the maximum subcarrier constraint considers the maximum capacity of a wavelength channel is limited by the all-optical reach. But the modulation format issue is not drawn in this work. Another path-based ILP model was presented in [8] to solve static RMLSA problem. In the model, candidate paths for each source-destination pair are pre-defined. The demand can be routed on only one of the candidate paths. The spectrum contiguity constraint is included in the ILP model, while the spectrum continuity constraint along a route is imposed implicitly since the assignment of a FS concerns the entire path. The objective is to minimize the number of FSs in the frequency spectrum that are assigned to at least one demand in the network.

Link-based ILP formulations of RSA can be viewed as a multi-commodity flow problem. Different from path-based model, there are no pre-computed candidate paths for each traffic request in link-based formulations. Hence, link-based schemes jointly decide the optimal routes and allocate spectra for all the services. Reference [9] proposed a linkbased ILP model (referred to as node-arc model) for RSA with the objective to minimize the maximal index of used FSs in the entire network. To reduce the number of variables and computational complexity, the model adopted the method in [4] to define each spectrum path by using the starting and ending FS indexes. The spectrum contiguity, spectrum contiguity, non-overlapping constraints can be guaranteed in the node-arc model. Another link-based ILP model of RSA was studied in [10]. This work considers two objectives to minimize: the maximum FS index allocated on any fiber, and the total number of FSs over all fibers (summation of the maximum index of the sub-carriers over the fiber). Both [9] and [10] assumed the traffic demand in unit of FS, and the issue of modulation format decision was not considered. The model in [4] addressed the decision of modulation level. The flexibility is considerably limited due to the essence of path-based model. Once the $k$-shortest paths are computed, the modulation level of a path is 
determined. The authors in reference [11] proposed a linkbased MILP formulation to design an EON with the consideration on multiple modulation formats and noise constraint. It is assumed that the optical transmission reach of high-bit-rate lightpaths is severely limited by the signal quality degradation. Hence, the choice of modulation format depends on the threshold on the cumulative spontaneous emission noise power of the lightpath with specific modulation format. Comparing to the path-based models, more variables and constraints are needed for link-based models to determine optimal routing path. This may significantly increase the complexity of optimization models. However, link-based models can achieve better performance because no pre-determined routing paths means more feasible path candidates and spectrum allocation decisions can be searched.

In both traditional and elastic optical networks, the signal impairment is accumulated along the path which it traverses. Especially, a higher-data-rate signal can traverse a shorter distance than a lower-data-rate signal before the quality of signal is deteriorated to an unacceptable level. To keep the signal quality acceptable, the optical transmission reach must be considered to guarantee QoT. Reference [12] proposed energy efficient network design for the mixed-line-rate IP over WDM networks with limited transmission reaches. In the context of EON, [11] and [13] considered the physical layer constraint during network design and planning. In [11], authors added the spontaneous emission noise into the constraints of MILP model. As multiple modulation formats are available in EON, the different signal-to-noise rate requirements of different modulation formats should be considered during solving RMLSA problem. Reference [13] defined a physical feasibility function which identified the feasible transmission reach, given the rate, and spectrum or modulation format used for a specific BVT.

In this paper, we present a link-based MILP model to solve the RMLSA problem in EON with the constraint of optical transmission reach. The contribution of our proposed model, compared to previous works, is as follows: (1) as a unneglectable key factor during modulation decision procedure, the transmission reaches of different modulation formats are considered as constraint for RMLSA problem, which is reasonable and practical; (2) modulation format is determined jointly with routing and spectrum allocation according to the given required data rates for requests. This procedure reflects the essence and advantage of EON; (3) spectrum continuity constraint and contiguity constraint are included while determining suitable modulation format and number of FSs.

\section{MATHEMATICAL Model}

\section{A. Problem Statement}

In OFDM-based EON, the traffic demand between source and destination node pair is transmitted over multiple lowrate subcarriers which are orthogonal to each other. Given the physical topology, the traffic matrix, and the limit of transmission reach for various modulation formats as the input, we need to:
- provision all source-destination connection requests: determine the route, appropriate modulation level and spectrum allocation for each traffic demand

- optimize the spectrum utilization: minimize the sum of the maximum FS index over all fibers in network

The following assumptions are stated in the RMLSA model: (a) The fiber capacity in terms of FSs is not limited on all links; (b) Connection requests are directional, and an end-to-end all-optical path must be found for each request; (c) No specific path for a connection is given in advance, i.e., any possible path and any possible set of contiguous FSs will be evaluated while solving the model. Fig. 1 (a) shows a linear network, from node A to node E. The spectral resource on each optical fiber is divided into FSs with the granularity of $12.5 \mathrm{GHz}$. Three modulation formats can be supported: BPSK, QPSK, and 8QAM. The maximum transmission reaches of optical signal are: $2000 \mathrm{~km}$ (BPSK), $1000 \mathrm{~km}$ (QPSK), and $500 \mathrm{~km}$ (8QAM). The request is represented by three tuples: $(s, d, B W)$, including source node $s$, destination node $d$, and required bandwidth/data rate $B W$. In the illustrative example, there are three connection requests: $R_{I}$ (A, C, $\left.50 \mathrm{Gbps}\right), R_{2}\left(\mathrm{~B}, \mathrm{D}, 35 \mathrm{Gbps}\right.$ ), and $R_{3}$ (A, $\mathrm{E}, 100 \mathrm{Gbps})$. To establish lightpath for $R_{l}$, the modulation format should be determined by considering path length (AB-C, $700 \mathrm{~km}$ ). Among three modulation formats, BPSK and QPSK can satisfy this requirement. We chose QPSK which can achieve higher spectrum efficiency, and allocate two FSs to $R_{l}$. For $R_{2}$, path length is $500 \mathrm{~km}$, and all three modulation formats are available. 8QAM is determined for the lightpath (B-C-D, $500 \mathrm{~km}$ ) and $1 \mathrm{FSs}$ are allocated to $R_{2}$. For $R_{3}$, path length is $1300 \mathrm{~km}$. Only BPSK can satisfy the QoT requirement. Hence, BPSK is determined for the lightpath (A-B-C-D-E), and 4 FSs are allocated to $R_{3}$. Fig. 1 (b) shows the spectrum allocation on each link, which satisfies the spectrum continuity and contiguity constraint. In Fig. 1 (b), FSs from index 1 to 2 on link A-B and B-C are allocated to $R_{1}$. FS 5 on link B-C and C-D are allocated to $R_{2}$. FS 8 to 11 on link A-B, B-C, C-D and D-E are allocated to $R_{3}$. The guard band between two lightpaths which share a common link is 2 FSs, e.g., FS 3 to 4,6 to 7 on link B-C, FS 6 to 7 on link C-D.

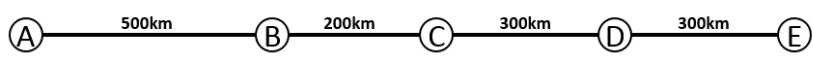

(a) Network topology

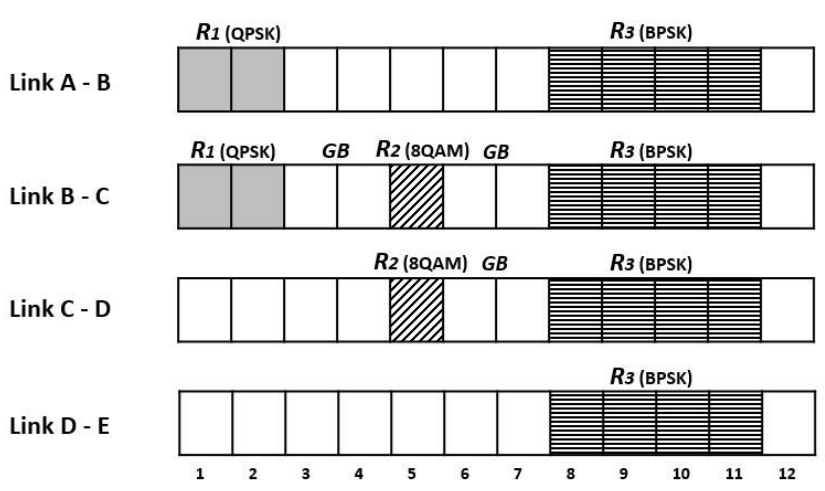

(b) Spectrum allocation on each link

Fig. 1 RMLSA for five-node linear network 


\section{B. MILP Formulation}

The notations and formulation are summarized as follows:

\section{Indexing rules:}

$(s, d)$ - source-destination pair, $s$ and $d$ represent the originating and terminating nodes of a connection request.

$(m, n)$ - node pair, $m$ and $n$ represent two endpoints of a fiber link in physical topology. Note that link has no directionality, i.e., $(m, n)$ and $(n, m)$ represent the same link in physical topology.

\section{Given:}

$G(V, E)$ - Network physical topology consisting of node set $V$ and edge set $E$

$\lambda^{s d}$ - Demand of connection request from $s$ to $d, s, d \in V$

$T M=\left[\lambda^{s d}\right]-$ Traffic matrix

$C$ - Base capacity of a FS with single bit per symbol modulation (BPSK)

$R$ - Set of modulation levels, $R=\{1,2,3, \ldots\}, r \in R$

$L_{m n}$ - Length of physical link $(m, n)$

$L_{r}$ - Maximum optical transmission reach of a lightpath adopting modulation level $r$

$G B$ - Number of FSs for filter guard band

$N E_{i}$ - Set of edges which are connected with node $i$

\section{Variables:}

$N_{s d, r}$ (integer) Number of FSs allocated to serve request $(s, d)$ with modulation level $r$

$N F S_{s d}$ - (integer) Number of FSs allocated to serve request $(s, d)$

$N l_{m n}^{s d, r}$ - (integer) Number of FSs allocated to serve request $(s, d)$ with modulation level $r$ on physical link $(m, n)$

$S_{s d}$ - (integer) Index of the starting FS allocated to serve request $(s, d)$

$E_{s d}$ - (integer) Index of the ending FS allocated to serve request $(s, d)$

$X_{s^{\prime} d^{\prime}}^{s d}$ - (binary) equals one if the ending FS index of the lightpath between pair $\left(s^{\prime}, d^{\prime}\right)$ is smaller than the starting FS index of the lightpath between $(s, d)$, i.e., $S_{s d}>E_{s^{\prime}} d^{\prime}$; otherwise, it takes the value of zero if the ending FS index of the lightpath between $(s, d)$ pair is smaller than the starting FS index of the lightpath between $\left(s^{\prime}, d^{\prime}\right)$, i.e., $S_{s^{\prime} d^{\prime}}>E_{s d}$. This variable indicates the relative positions of the FSs allocated for two lightpaths.

$Y_{m n}^{s d, r}$ - (binary) equals one if request $(s, d)$ using modulation level $r$ is routed on physical link $(m, n)$; otherwise, it takes value of zero.

$Z_{s d, r^{-}}$(binary) equals one if modulation level $r$ is determined for connection request $(s, d)$; otherwise, it takes value of zero.

$P N_{i}^{s d, r}$ - (binary) equals one if connection request $(s, d)$ using modulation level $r$ bypasses node $i$; otherwise, it takes value of zero.

$M I_{m n}$ - (integer) Maximum index of FS allocated on physical link $(m, n)$

\section{Objective function:}

$$
\text { Minimize } \sum_{(m, n) \in E} M I_{m n}
$$

The objective is to minimize the sum of the maximum index of allocated FSs on all links in the entire network

\section{Constraints:}

1) Single path routing constraint:

$$
\begin{gathered}
\sum_{(m, n) \in N E_{s}} Y_{m n}^{s d, r}=1, \forall(s, d), \forall r \in R \\
\sum_{(m, n) \in N E_{d}} Y_{m n}^{s d, r}=1, \forall(s, d), \forall r \in R \\
\sum_{(m, n) \in N E_{i}} Y_{m, ~}^{s d, r}=2 \times P N_{i}^{s d, r}, \forall(s, d), \forall r \in R, \forall i \in V, i \neq s, d \\
P N_{i}^{s d, r}+P N_{j}^{s d, r} \geq 2 \times Y_{m n}^{s d, r}, \forall(s, d), \forall r \in R, \forall(m, n) \in E
\end{gathered}
$$

Constraint (2)-(5) guarantee that for each request $(s, d)$, only one single path can be followed, and the traffic demand cannot be split into multiple flows. Equation (2) regulates that the first-hop physical link on the lightpath for each connection request $(s, d)$ must start from node $s$. Hence, the sum of all links going out from source node $s$ must be equal to one. Equation (3) ensures that the last-hop physical link on the lightpath for each connection request $(s, d)$ must end at node $d$. Similarly, the sum of all links coming in to destination node $d$ must be equal to one. Equation (4) limits that for any intermediate node traversed by a lightpath, there must be two physical links connected with the node (one injecting into and one ejecting out from the node). Equation (5) ensures that if any physical link $(m, n)$ is traversed by a lightpath $(s, d)$, the two ends (node $\mathrm{m}$ and $\mathrm{n}$ ) of the link must be traversed by the lightpath as well.

$$
\begin{gathered}
\text { 2) Flow conservation constraint: } \\
\sum_{(m, n) \in N E_{s}} N l_{m n}^{s d, r}=N_{s d, r}, \forall(s, d), \forall r \in R \\
\sum_{(m, n) \in N E_{d}} N l_{m n}^{s d, r}=N_{s d, r}, \forall(s, d), \forall r \in R \\
\sum_{(m, n) \in N E_{i}} N l_{m n}^{s d, r}=2 \times N_{s d, r}, \forall(s, d), \forall r \in R, \forall i \in V, i \neq s, d
\end{gathered}
$$

Constraint (6), (7), and (8) ensures the flow conservation in terms of number of FSs for any request $(s, d)$. Equation (6) regulates that for the number of FSs allocated on the first-hop physical link of the lightpath $(s, d)$ must equals to the number of FSs allocated to connection request $(s, d)$, under any modulation level $r$. Equation (7) regulates that for the number of FSs allocated on the last-hop physical link of the lightpath $(s, d)$ must equals to the number of FSs allocated to connection request $(s, d)$, under any modulation level $r$. Equation (8) ensures that for any intermediate node $i$ on the lightpath $(s, d)$, the number of FSs allocated on the incoming and outgoing physical links must equals to twice the number of FSs required for request $(s, d)$ under any modulation level $r$.

3) Starting Frequencies ordering constraint:

$$
X_{s^{\prime} d^{\prime}}^{s d}+X_{s d}^{s^{\prime} d^{\prime}}=1, \forall(s, d),\left(s^{\prime}, d^{\prime}\right): s d \neq s^{\prime} d^{\prime}
$$

Constraint (9) ensures that either the starting FS of $(s, d)$ 
is smaller than ending FS of $\left(s^{\prime}, d^{\prime}\right)$, or the starting FS of $\left(s^{\prime}\right.$, $\left.d^{\prime}\right)$ is smaller than ending FS of $(s, d)$. The relative position of two connection requests is determined by $X_{s^{\prime} d^{\prime}}^{s d}$. If $X_{s^{\prime} d^{\prime}}^{s d}$ equals one (i.e., $S_{s d}>E_{s^{\prime} d^{\prime}}$ ), then $X_{s d}^{s^{\prime} d^{\prime}}$ must be zero; vice versa.

4) Spectrum continuity and non-overlapping spectrum allocation constraints:

$$
\begin{array}{r}
E_{s^{\prime} d^{\prime}}-S_{s d} \leq F \times\left(X_{s^{\prime} d^{\prime}}^{s d}+2-Y_{m n}^{s d, r}-Y_{m n}^{s^{\prime} d^{\prime}, r^{\prime}}\right)-G B-1, \\
\forall(s, d),\left(s^{\prime}, d^{\prime}\right): s d \neq s^{\prime} d^{\prime}, \forall(m, n) \in E
\end{array}
$$

Constraint (9) and (10) ensure that when two lightpaths share common link(s) they must not overlap in frequency domain. In detail, when two lightpaths share a common link $(m, n)\left(Y_{m n}^{s d, r}=Y_{m n}^{s^{\prime} d^{\prime}, r^{\prime}}=1\right)$ and $S_{s^{\prime} d^{\prime}}>E_{s d}\left(X_{s^{\prime} d^{\prime}}^{s d}=0\right)$, we can obtain $E_{s^{\prime} d^{\prime}}+G B+1 \leq S_{s d}$, which satisfies the nonoverlapping constraint; otherwise, constraint (10) always holds.

\section{5) Physical Link Usage Constraint: $N l_{m n}^{s d, r} \leq Y_{m n}^{s d, r} \cdot F, \quad \forall(s, d), \forall(m, n) \in E, \forall r \in R$}

Constraint (11) ensures that only the FSs on those links $(m, n)$ which are used to route request $(s, d)$ can be allocated. For any physical link $(m, n)$ if $Y_{m n}^{s d, r}$ equals zero, i.e. the link is not traversed by lightpath $(s, d)$, then the number of FSs on link $(\mathrm{m}, \mathrm{n})$ allocated to lightpath $(s, d)$ must be zero $\left(N l_{m n}^{s d, r}=0\right)$. In contrast, if physical link $(m, n)$ is traversed by lightpath $(s, d)\left(Y_{m n}^{s d, r}=1\right)$, the number of FSs on link ( $m$, $n)$ which can be allocated to lightpath $(s, d)$ is less than a very large value $F\left(N l_{m n}^{s d, r} \leq F\right.$ always holds when $\left.Y_{m n}^{s d, r}=1\right)$.

\section{6) Spectrum path MF constraint:}

$$
N_{s d, r} \leq Z_{s d, r} \cdot F, \quad \forall(s, d), \forall r \in R
$$

Constraint (12) determines which of the modulation level is used for the spectrum path between $(s, d)$ pair. If $Z_{s d, r}=0$, that is, modulation level $r$ is not adopted by connection request $(s, d)$, the number of FSs allocated to serve request $(s, d)$ with modulation level $r$ must be zero $\left(N_{s d, r}=0\right)$. In contrast, if modulation level $r$ is adopted by connection request $(s, d)$, the number of FSs allocated to serve request $(s, d)$ under modulation level $r$ is smaller than a very large value $F\left(N_{s d, r} \leq F\right.$ always holds when $\left.Z_{s d, r}=1\right)$.

\section{7) Modulation Format Constraint:}

$$
\sum_{r \in R} Z_{s d, r}=1, \quad \forall(s, d)
$$

Constraint (13) asserts that each lightpath can adopt only one modulation level. For any connection request $(s, d)$, the sum of modulation levels of lightpath must be equal to one.

\section{8) Transmission reach constraint:}

$$
\sum_{(m, n) \in E} Y_{m n}^{s d, r} \cdot L_{m n} \leq L_{r}, \quad \forall(s, d), \forall r \in R
$$

Constraint (14) ensures that the path length of any request $(s, d)$ adopting modulation level $r$ cannot greater than the maximum optical transmission reach under $r$. The path length is the sum of distance of all physical links which are traversed by the lightpath $(s, d)$.

9) Max_FS_ID Constraint:

$$
\begin{aligned}
M I_{m n} \geq E_{s d}-F \cdot & \left(1-Y_{m n}^{s d, r}\right), \\
& \forall(s, d), \forall(m, n) \in E, \forall r \in R
\end{aligned}
$$

Constraint (15) ensures that for any physical link $(m, n)$ $M I_{m n}$ is greater than the ending FS index of any request $(s$, d) which is routed on link $(m, n)$.

10) Others:

$$
N_{s d, r}=Z_{s d, r} \times\left\lceil\lambda_{s d} / r \cdot C\right\rceil, \forall(s, d), \forall r \in R
$$

Equation (16) calculates the number of FSs for each $(s, d)$ with modulation level $r$ :

$$
E_{s d}=S_{s d}+N F S_{s d}-1, \forall(s, d)
$$

Equation (17) calculates the ending FS index for each request $(s, d)$.

$$
N F S_{s d}=\sum_{r \in R} N_{s d, r}, \quad \forall(s, d)
$$

Equation (18) calculates the number of FSs allocated for each request $(s, d)$.

\section{NumericAl RESUlTS}

To evaluate the performance of the proposed MILP model, we apply it on case study. The numerical results will be shown and analyzed in this section. Our results are obtained via optimization software IBM ILOG CPLEX Optimization Studio Version12.6 on the computer with Intel Core (TM) i5-2500 CPU (3.30 GHz) and 8 GB RAM.

The case study is implemented on a four-node (4N4L) ring network, and a six-node nine-link (6N9L) mesh network as shown in Fig. 2 and Fig. 3, respectively. Nodes are connected by bi-directional links. The filter guard band is $G B=2$ FSs. The traffic demands for each source-destination pair is randomly generated between 1 and $D$ Gbps $(D=100$, $150,200,250,300,400)$. The bandwidth of one FS is $C=$ $12.5 \mathrm{GHz}$.

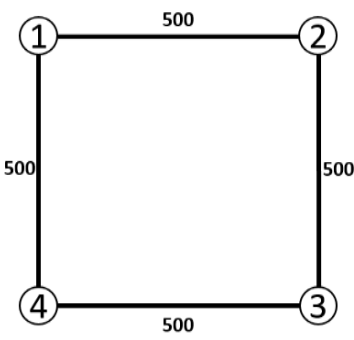

Fig. 2 Four-node ring network topology

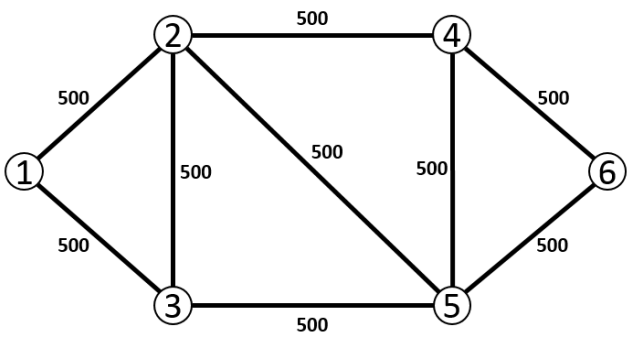

Fig. 3 Six-node nine-link network topology 
Three modulation formats are considered: BPSK, QPSK, and 8QAM, and the corresponding modulation levels are 1, 2 , and 3. The parameters for different modulation formats are summarized in Table I.

TABLE I

PARAMETERS FOR DIFFERENT MODULATION FORMAT

\begin{tabular}{cccc}
\hline \hline $\begin{array}{c}\text { Modulation } \\
\text { Format }\end{array}$ & $\begin{array}{c}\text { Spectrum } \\
\text { Efficiency } \\
\text { (bps/Hz) }\end{array}$ & $\begin{array}{c}\text { Data Rate per } \\
\text { Subcarrier } \\
\text { (Gbps) }\end{array}$ & $\begin{array}{c}\text { Transmission } \\
\text { Reach }(\mathbf{k m})\end{array}$ \\
\hline BPSK & 1 & 12.5 & 2000 \\
QPSK & 2 & 25 & 1000 \\
8QAM & 3 & 37.5 & 500 \\
\hline \hline
\end{tabular}

Fig. 4 shows the number of required FSs to accommodate all traffic in 4N4L ring network according to different values of $D$ (large value of $D$ represents heavy traffic load). As a reference, we evaluate the scenario that only the modulation format BPSK is supported to quantify the spectrum usage affected by flexible choice of multiple modulation levels.

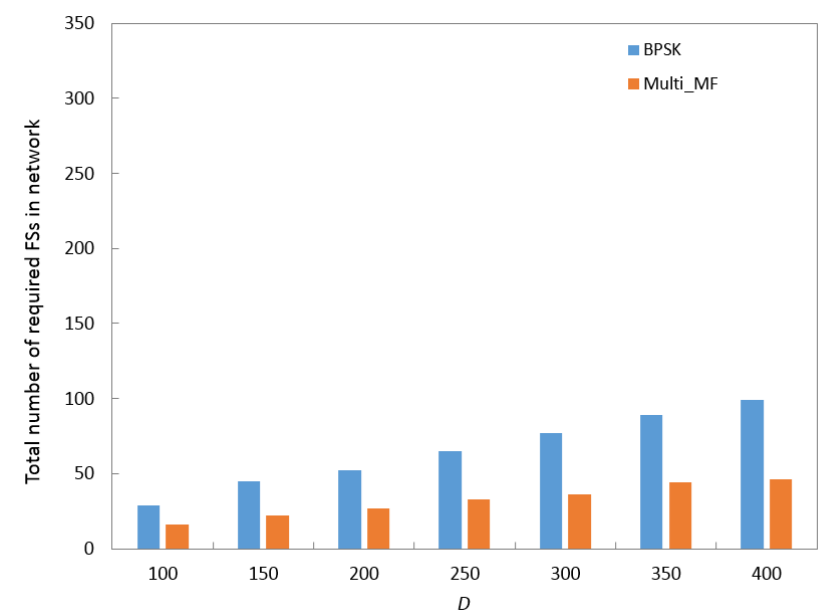

Fig. 4 Total number of required FSs in network according to different $D$ for the 4N4L ring topology

The group of orange bars indicates the results for the case with multiple modulation formats, shorted as "Multi-MF". The group of blue bars indicates the results for the case with single modulation format, shorted as "BPSK". Obviously, the number of required FSs of Multi-MF is much less than that of BPSK under all different $D$. Multi-MF can achieve $45-53 \%$ saving on spectrum usage in terms of required FSs, comparing to the BPSK case. In addition, the saving on spectrum resources is improved with increasing network traffic load. These observations indicate that to accommodate the same network load, Multi-MF can outperform BPSK by efficiently allocating spectrum resources while satisfying the requirement of QoT.

Table II and III show the maximum index of FS on all links of 4N4L ring network based on Multi-MF and BPSK, respectively. It can be found that the difference among the maximum FS indexes of links is larger based on BPSK than that based on Multi-MF. For example, when $D=100 \mathrm{Gbps}$, the difference is 9 FSs between the links with the smallest and the largest indexes under Multi-MF while the difference increases to $11 \mathrm{FSs}$ under BPSK. Because the number of required FSs is determined solely on the bandwidth of request in the single MF scenario, the benefit of high spectral efficiency from OFDM cannot be exploited. Again, this difference becomes larger according to increasing the traffic load. Under $D=400 \mathrm{Gbps}$, the variation on the maximum FS indexes among all links based on Multi-MF and BPSK is 16 and 38, respectively. Larger variation indicates heavier load unbalance brought by BPSK scheme because the rigidness on allocate appropriate number of FSs.

TABLE II

MAXIMUM INDEX OF FS ON LINKS (MULTI-MF) FOR 4N4L NETWORK

\begin{tabular}{|c|c|c|c|c|c|c|c|}
\hline$\underbrace{}_{\operatorname{Link}} D$ & 100 & 150 & 200 & 250 & 300 & 350 & 400 \\
\hline $1->2$ & 0 & 0 & 11 & 9 & 14 & 12 & 12 \\
\hline $1->4$ & 3 & 4 & 7 & 13 & 10 & 16 & 17 \\
\hline $2->3$ & 4 & 6 & 4 & 0 & 5 & 1 & 1 \\
\hline $3->4$ & 9 & 12 & 5 & 11 & 7 & 15 & 16 \\
\hline
\end{tabular}

TABLE III

MAXIMUM INDEX OF FS ON LINKS (BPSK) FOR 4N4L NETWORK

\begin{tabular}{|c|c|c|c|c|c|c|c|}
\hline$\overbrace{\operatorname{Link}} D$ & 100 & 150 & 200 & 250 & 300 & 350 & 400 \\
\hline $1->2$ & 7 & 11 & 18 & 15 & 18 & 21 & 23 \\
\hline $1->4$ & 11 & 16 & 12 & 22 & 26 & 29 & 32 \\
\hline $2->3$ & 0 & 1 & 7 & 2 & 2 & 3 & 3 \\
\hline $3->4$ & 11 & 17 & 15 & 26 & 31 & 36 & 41 \\
\hline
\end{tabular}

Fig. 5 shows the number of required FSs to accommodate all traffic as a function of different traffic load for the 6N9L mesh network. In general, Multi-MF obtains much less number of FSs than that of BPSK under all different traffic loads. Multi-MF can achieve $36-47 \%$ saving on spectrum usage in terms of required FSs, comparing to the BPSK. In addition, the saving on FSs enlarges according to increasing the traffic load.

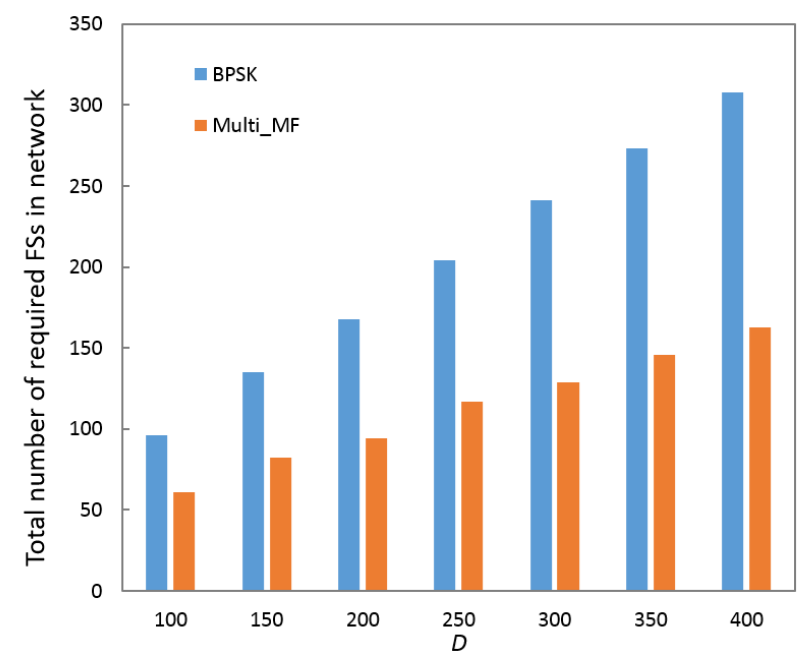

Fig. 5 Total number of required FSs in network according to different $D$ for the 6N9L mesh topology

Table IV and $\mathrm{V}$ show the maximum index of FS on all links of 6N9L mesh network based on Multi-MF and BPSK, respectively. The variation on the maximum indexes of FSs which are allocated on all links is much larger based on BPSK than that of Multi-MF. Under low traffic load $D=$ 
100 , the FS index variation is 13 based on Multi-MF. While the variation increases to $35 \mathrm{FSs}$ based on BPSK. Under high traffic load $D=400$, the FS index variation is 54 based on BPSK. While the variation based on Multi-MF is only 23 , even smaller than the half of that based on BPSK. Several traditional RMLSA schemes assume single MF (higher spectral efficiency compared to WDM networks, e.g., $\mathrm{ML}=3$ or 4 ) for simplicity, which ignores the nature of flexibility in EON. Hence this assumption is not practical.

TABLE IV

MAXIMUM INDEX OF FS ON LINKS (MULTI-MF) FOR 6N9L NETWORK

\begin{tabular}{|c|c|c|c|c|c|c|c|}
\hline$\underbrace{}_{\operatorname{Link}} D$ & 100 & 150 & 200 & 250 & 300 & 350 & 400 \\
\hline $1->2$ & 12 & 12 & 17 & 6 & 24 & 22 & 24 \\
\hline $1->3$ & 0 & 4 & 1 & 20 & 1 & 6 & 6 \\
\hline $2->3$ & 5 & 6 & 8 & 9 & 7 & 12 & 14 \\
\hline $2->4$ & 13 & 14 & 10 & 13 & 20 & 26 & 29 \\
\hline $2->5$ & 10 & 12 & 24 & 13 & 23 & 22 & 24 \\
\hline $3->5$ & 5 & 10 & 8 & 25 & 15 & 17 & 19 \\
\hline $4->5$ & 2 & 6 & 5 & 6 & 10 & 8 & 10 \\
\hline $4->6$ & 6 & 8 & 0 & 0 & 10 & 11 & 13 \\
\hline $5->6$ & 8 & 10 & 21 & 25 & 19 & 22 & 24 \\
\hline
\end{tabular}

TABLE V

MAXIMUM INDEX OF FS ON LINKS (BPSK) FOR 6N9L NETWORK

\begin{tabular}{|c|c|c|c|c|c|c|c|}
\hline$\overbrace{\operatorname{Link}} D$ & 100 & 150 & 200 & 250 & 300 & 350 & 400 \\
\hline $1->2$ & 12 & 20 & 13 & 27 & 13 & 30 & 22 \\
\hline $1->3$ & 4 & 3 & 12 & 4 & 23 & 12 & 23 \\
\hline $2->3$ & 10 & 14 & 19 & 19 & 27 & 31 & 36 \\
\hline $2->4$ & 12 & 25 & 20 & 20 & 27 & 30 & 36 \\
\hline $2->5$ & 19 & 22 & 30 & 46 & 37 & 57 & 54 \\
\hline $3->5$ & 14 & 15 & 29 & 29 & 47 & 39 & 53 \\
\hline $4->5$ & 7 & 11 & 15 & 23 & 22 & 26 & 30 \\
\hline $4->6$ & 0 & 10 & 0 & 0 & 0 & 0 & 0 \\
\hline $5->6$ & 18 & 15 & 30 & 36 & 45 & 48 & 54 \\
\hline
\end{tabular}

\section{CONCLUSION}

In recent years, OFDM-based EON has been extensively studied as a promising paradigm which has high flexibility in traffic accommodation and can improve spectrum utilization. We proposed an MILP model which addresses the essential RMLSA issue in EON, focusing on determining appropriate modulation format and number of FSs for each request with the consideration of QoT. The numerical results showed that the proposed model could reduce the number of FSs required in network, comparing to the schemes which assume a fixed modulation format. Moreover, multiple modulation formats could help alleviate the uneven consumption of spectrum among fiber links.

Since the RMLSA problem is NP-hard and the computational complexity of optimization model is too high, we consider developing heuristic algorithms to solve the RMLSA problem in large-size networks. In addition, the energy efficiency, protection, fragmentation issues in EON can be studied for future work.

\section{ACKNOWLEDGMENT}

This work was supported by Basic Research Program through the National Research Foundation of Korea (NRF) funded by the Ministry of Education (2014-055177).

\section{REFERENCES}

[1] S.K. Korotky, "Traffic trends: Drivers and measures of cost-effective and energy-efficient technologies and architectures for backbone optical networks," Optical Fiber Communication Conference and Exposition and the National Fiber Optic Engineers Conference (OFC/NFOEC), Los Angeles, CA, 2012.

[2] O. Gerstel, "Elastic optical networking: a new dawn for the optical layer?," IEEE Communications Magazine, vol.50, no.2, pp.s12-s20, February 2012.

[3] F. Tang, "Electronic Traffic Grooming in Dedicated Path Protected IP over Elastic Optical Network," Asia Communication and Photonics Conference (ACP), 2015.

[4] K. Christodoulopoulos, I. Tomkos, E. Varvarigos, "Routing and spectrum allocation in OFDM-based optical networks with elastic bandwidth allocation," in: Proceedings of IEEE Globecom, 2010.

[5] K. Christodoulopoulos, "Elastic Bandwidth Allocation in Flexible OFDM-Based Optical Networks," Journal of Lightwave Technology, vol.29, no.9, pp.1354-1366, May. 2011.

[6] W. Wei, C. Wang, X. Liu, "Adaptive IP/optical OFDM networking design," Conference on Optical Fiber Communication, collocated National Fiber Optic Engineers Conference (OFC/NFOEC), vol. 1, no. 3, pp.21-25, March 2010.

[7] A. Nag, T. Wang, B. Mukherjee, "On Spectrum-Efficient Green Optical Backbone Networks," IEEE Global Telecommunications Conference (GLOBECOM), vol. 1, no. 5, pp.5-9, Dec. 2011.

[8] M. Klinkowski, K. Walkowiak, "Routing and Spectrum Assignment in Spectrum Sliced Elastic Optical Path Network," IEEE Communications Letters, vol.15, no.8, pp.884-886, August 2011.

[9] A. Cai, "Novel Node-Arc Model and Multi-Iteration Heuristic for Static Routing and Spectrum Assignment in Elastic Optical Networks," IEEE/OSA Journal of Lightwave Technology, Vol. 31, No. 21, pp. $3402-3413$. Sept. 2013.

[10] Y. Wang, "Towards elastic and fine-granular bandwidth allocation in spectrum-sliced optical networks," Journal of Optical Commun Communications and Networking, Vol. 4, Issue 11, pp. 906-917, 2012.

[11] K.D.R. Assis, R.C. Almeida, A.V.T. Cartaxo, A.F. dos Santos, H Waldman, "Flexgrid optical networks design under multiple modulation formats," International Telecommunications Symposium (ITS), vol. 1, no. 5, pp.17-20, Aug. 2014.

[12] Y. Lui, G. Shen, W. Shao, and S. K. Bode, "Green IP over WDM Optical Network Design for Mixed Line Rates and Limited Transmission Reaches," in Proc. COIN 2013.

[13] K. Christodoulopoulos, P. Soumplis, and E. Varvarigos. "Planning Flexgrid Optical Networks under Physical Layer Constraints" Journal of Optical Communications and Networking, Vol. 5, Issue 11, pp. 1296-1312, 2013. 